6. M. Riesz, Sur la sommation des séries de Dirichlet, C. R. Acad. Sci. Paris vol. 149 (1909) pp. 18-21.

7. - Sur l'équivalence de certaines méthodes de sommation, Proc. London Math. Soc. (2) vol. 22 (1923-1924) pp. 412-419.

8. L. L. Silverman, On the definition of the sum of a divergent series, University of Missouri Studies, Mathematical Series, vol. 1, no. 1, 1913.

9. O. Toeplitz, Über allgemeine lineare Mittelbildungen, Prace Matematycznofizyczne vol. 22 (1911) pp. 113-119.

The UnIVersity of Rochester

\title{
ON THE REPRESENTATION OF A FUNCTION AS A HELLINGER INTEGRAL
}

RICHARD H. STARK

We derive in this note a necessary and sufficient condition that a nondecreasing, continuous function $h$ of a single variable $x$ be representable as a Hellinger integral of the form $\int_{0}^{x}(d f)^{2} / d g$. This condition was first proved by Hellinger in his dissertation [1]. ${ }^{1}$ Other proofs have been given by Hahn [2] and Hobson [3], who transform to Lebesgue integrals and make use of Lebesgue theory. Hellinger's proof and the less complicated proof given here have a certain simplicity in that they avoid reliance on these notions and even remain entirely within the range of monotone functions.

We consider nondecreasing functions of a real variable $x$ on the interval $0 \leqq x \leqq 1$ (henceforth denoted as $[0,1]$ ). For such a function $f(x)$ and a closed interval $\Delta$ with end points $x_{1}$ and $x_{2}\left(x_{1} \leqq x_{2}\right)$, we define a new function $f_{\Delta}(x)$ to denote the length of the interval on the $f$-axis determined by the interval on the $x$-axis common to $\Delta$ and $(0, x)$. More precisely, denoting

$$
\begin{aligned}
& f(x \pm 0)=\lim _{h \rightarrow 0} f(x \pm|h|) \quad \text { if } 0<x<1, \\
& f(0-0)=f(0) ; \quad f(1+0)=f(1),
\end{aligned}
$$

we define

$$
f_{\Delta}(x)= \begin{cases}0 & \text { if } 0 \leqq x<x_{1}, \\ f(x+0)-f\left(x_{1}-0\right) & \text { if } x_{1} \leqq x \leqq x_{2}, \\ f\left(x_{2}+0\right) & \text { if } x_{2}<x \leqq 1 .\end{cases}
$$

Received by the editors January 30, 1948.

1 Numbers in brackets refer to the references cited at the end of the paper. 
The interval $\left[f\left(x_{1}-0\right), f\left(x_{2}+0\right)\right]$ on the $f$-axis will be denoted as $f(\Delta)$. We retain the notation $\Delta f$ for the proper difference $f\left(x_{2}\right)-f\left(x_{1}\right)$.

Given sets $\left\{E_{i}\right\} \subset[0,1]$ for which the symbols $f_{E_{i}}(x)$ and $f\left(E_{i}\right)$ have been defined, we define

$$
\begin{aligned}
& \left.\begin{array}{l}
f \Sigma E_{i}(x)=\sum f_{B_{i}}(x) \\
f\left(\sum E_{i}\right)=\sum f\left(E_{i}\right)
\end{array}\right\} \quad \text { if } E_{i} \cdot E_{j}=0 \text { when } i \neq j . \\
& \left.f_{E_{2}-E_{1}}(x)=f_{E_{2}}(x)-f_{E_{1}}(x)\right) \\
& \left.f\left(E_{2}-E_{1}\right)=f\left(E_{2}\right)-f\left(E_{1}\right)\right\} \\
& \text { if } E_{1} \subset E_{2} \text {. }
\end{aligned}
$$

The sets which we shall consider will be constructed from sets of intervals in a manner such that (1) and (2) will assign to each such set $E$ a function $f_{E}(x)$ called the measure function of $E$ on the $f$-axis and a set $f(E)$ of points on the $f$-axis. Our $f_{E}(1)$ is just the measure with respect to $f(x)$ of the set $E$ as defined in $[4 ;$ p. 277]. It is immediate that if $f_{E_{D}}(x)$ denotes the measure on the $f_{E}(x)$ axis of the set $D$ on the $x$-axis, then

$$
f_{E D}(x)=f_{E \cdot D}(x) .
$$

We now consider functions $f(x), g(x)$, and $h(x)$ which are nondecreasing and continuous in $[0,1]$ and satisfy the inequality

$$
(\Delta f)^{2} \leqq \Delta g \Delta h \quad \text { on every subinterval } \Delta \text { of }[0,1] .
$$

Let $M$ be an arbitrary set on the $x$-axis. For arbitrary $\epsilon>0$, the set $g(M)$ on the $g$-axis may be enclosed in a set $I_{\mathrm{e}}$ of countably many mutually disjoint intervals $d_{i}$ such that the measures on the $g$-axis of the sets $g(M)$ and $I_{\epsilon}$ differ by less than $\epsilon$; that is, the inverse function $x(g)$ defines a corresponding set $x\left(I_{6}\right)$ of the $x$-axis which we denote for brevity as $X_{\mathbf{t}}$ such that

$$
0 \leqq g_{X_{\epsilon}}(1)-g_{M}(1) \leqq \epsilon .
$$

Now for $\Delta$ an arbitrary interval of the $x$-axis and $\Delta_{i}$ the intersection of $\Delta$ with $x\left(d_{i}\right)$, we have:

$$
\Delta f_{M} \leqq \Delta f_{X_{e}}=\sum_{i=1}^{\infty} \Delta_{i} f .
$$

But $\left(\Delta_{i} f\right)^{2} \leqq \Delta_{i} g \Delta_{i} h$ on every $\Delta_{i}$ so that from the Cauchy-Schwarz inequality:

$$
\left(\sum_{i=1}^{\infty} \Delta_{i} f\right)^{2} \leqq \sum_{i=1}^{\infty} \Delta_{i} g \sum_{i=1}^{\infty} \Delta_{i} h \leqq \Delta g_{X_{\epsilon}} \Delta h .
$$


With use of (5) and (6), this gives

$$
\left(\Delta f_{M}\right)^{2} \leqq \Delta g_{M} \Delta h \text {. }
$$

Replacing (4) by (8) and carrying out an analogous argument for a set $N$ and the $h$-axis, we have

$$
\left(\Delta f_{M} \cdot N\right)^{2} \leqq \Delta g_{M} \Delta h_{N}
$$

if (4) holds.

TheOREM. To a given pair of nondecreasing, continuous functions $g(x)$ and $h(x)$ for which $g(0)=0=h(0)$ there corresponds an $f(x)$ such that

$$
h(x)=\int_{0}^{x} \frac{(d f)^{2}}{d g}
$$

if and only if for every set $E$ of the $x$-axis such that $g_{E}(1)=g(1)$, we have $h_{E}(1)=h(1)$.

For the proof, we use the following elementary properties of Hellinger integrals [1] which hold for arbitrary functions $g(x)$ and $h(x)$ that are nondecreasing and continuous in $[0,1]$ :

a. Existence of $t(x)=\int_{0}^{x}(d u)^{2} / d g$ implies $t(x)=\int_{0}^{x}\left(d \int_{0}^{x}(d g d t)^{1 / 2}\right)^{2} / d g$.

b. $f(x)=\int_{0}^{x}(d g d h)^{1 / 2}$ exists and $(\Delta f)^{2} \leqq \Delta g \Delta h$.

c. The inequality $(\Delta f)^{2} \leqq \Delta g \Delta h$ implies that $s(x)=\int_{0}^{x}(d f)^{2} / d g$ exists and $(\Delta f)^{2} / \Delta g \leqq \Delta s \leqq \Delta h$ if $\Delta g \neq 0$.

It follows from (10a) that the desired representation of $h(x)$ exists only if it is given by $\int_{0}^{x}(d f)^{2} / d g$ where $f(x)=\int_{0}^{x}(d g d h)^{1 / 2}$. By (10b), $(\Delta f)^{2} \leqq \Delta g \Delta h$. Let $E$ be any set such that $g_{E}(1)=g(1)$. Then from (8), we have on replacing $M$ by the complement $\bar{E}$ of $E$ with respect to $[0,1]$ and taking for $\Delta$ the interval $[0,1]$ that

$$
\left(f_{\bar{E}}(1)\right)^{2} \leqq g_{\bar{E}}(1) h(1)=0
$$

and consequently $f_{E}(1)=f(1)$. It follows from application of (11) to (9) that

$$
(\Delta f)^{2} \leqq \Delta g \Delta h_{E}
$$

Property (10c) with (12) gives that $\Delta s \leqq \Delta h_{E}$ so that the function $a(x)=h_{E}(x)-s(x)$ is nondecreasing and if $\Delta g \neq 0$

$$
\begin{aligned}
0 \leqq \Delta a & =\Delta h_{E}-\Delta s \leqq \Delta h-(\Delta f)^{2} / \Delta g \\
& =\left((\Delta g \Delta h)^{1 / 2}-\Delta f\right)\left((\Delta h / \Delta g)^{1 / 2}+\Delta f / \Delta g\right) .
\end{aligned}
$$

Consequently 


$$
\begin{aligned}
& 0 \leqq \Delta a=\Delta h_{E}-\Delta s \leqq 2(\Delta h / \Delta g)^{1 / 2}\left((\Delta g \Delta h)^{1 / 2}-\Delta f\right), \\
& \text { if } g_{E}(1)=g(1) \text { and } \Delta g \neq 0 .
\end{aligned}
$$

We next choose a sequence of divisions $D_{n}$ of the interval $(0,1)$ into finitely many nonoverlapping intervals (that is, no two intervals of $D_{n}$ have more than an end point in common) such that each $D_{n}$ is formed from $D_{n-1}$ by addition of finitely many division points and

$$
\sum_{D_{n}}\left((\Delta g \Delta h)^{1 / 2}-\Delta f\right) \leqq 4^{-n}
$$

That such a choice is possible is implied by (10a).

For each division $D_{n}$, we distinguish two types of intervals:

a. The set $G_{n}$ of intervals such that $\Delta g \geqq 4^{-n} \Delta h$;

b. The set $S_{n}$ of intervals such that $\Delta g<4^{-n} \Delta h$.

Then from (13), (14), (15), we have

$$
\begin{aligned}
& a_{G_{n}}(1)=\sum_{G_{n}} \Delta a \leqq 2^{-n+1}, \\
& g_{S_{n}}(1)=\sum_{S_{n}} \Delta g \leqq 4^{-n} h(1) .
\end{aligned}
$$

We define $R_{n}=\coprod_{m=n}^{\infty} G_{m}$ and $R=\lim _{n \rightarrow \infty} R_{n}$. It is immediate that

$$
a_{R}(1)=\lim _{n \rightarrow \infty} a_{R_{n}}(1) \leqq \lim _{n \rightarrow \infty} a_{G_{n}}(1)=0 \quad \text { (see (16)), }
$$

and since $\bar{R} \subset \sum_{m=n}^{\infty} S_{m}$,

$$
g_{\bar{R}}(1) \leqq \lim _{n \rightarrow \infty} \sum_{m=n}^{\infty} g_{S_{m}}(1)=0
$$

Thus $R$ is a set of the type $E$ assumed in (13). Consequently, $0 \leqq \Delta a$ $\leqq \Delta h_{R}$, and it follows from (9) and (3) that $a_{\bar{R}}(1) \leqq h_{R} \cdot \bar{R}(1)=0$. Hence

$$
a_{\bar{R}}(1)=0 \text {. }
$$

The function $a(x)$ is nondecreasing with $a(0)=0$ and by (18) and (19) has $a(1)=0$. Thus $a(x) \equiv 0$, that is,

$$
s(x)=h_{R}(x) .
$$

Therefore,

$$
s(x)=\int_{0}^{x} \frac{\left(d \int_{0}^{x}(d g d h)^{1 / 2}\right)^{2}}{d g}
$$

is the measure function on the $h$-axis of a set $R$ of the $x$-axis. This 
integral is actually equal to $h(x)$ if and only if $h_{R}(1)=h(1)$. The condition that $g_{E}(1)=g(1)$ implies $h_{E}(1)=h(1)$ is surely necessary to provide that $h(x)=s(x)$, for $g_{E}(1)=g(1)$ implies $s_{E}(1)=s(1)$. On the other hand, if $g_{E}(1)=g(1)$ does imply $h_{E}(1)=h(1)$, the condition that $h_{R}(1)=h(1)$ is fulfilled and $h(x) \equiv s(x)$. This completes the proof.

\section{REFERENCES}

1. E. D. Hellinger, Die Orthogonalinvarianten quadratischer Formen von unendlich vielen Veränderlichen, Inaugural-Dissertation, Göttingen, 1907.

2. Hans Hahn, Über die Integrale des Herrn Hellinger und die Orthogonalinvarianten der quadratischer Formen von unendlich vielen Veränderlichen, Monatshefte für Mathematik und Physik vol. 23 (1912) pp. 161-224.

3. E. W. Hobson, On Hellinger's integrals, Proc. London Math. Soc. vol. 18 (1919) pp. 249-265.

4. Henri Lebesgue, Lę̧ons sur l'intégration, Gauthier-Villars, Paris, 2d ed., 1928.

NORTHWESTERN UNIVERSITY 